\title{
Numerical analysis of geogrid contribution to railway track reinforcement
}

\author{
António Lanca ${ }^{1}$, Zuzana Dimitrovová ${ }^{1,2^{*}}$, Madalena Barroso $^{3}$ and Simona Fontul ${ }^{3}$ \\ ${ }^{1}$ DEC, Faculdade de Ciências e Tecnologia, Universidade Nova de Lisboa, Lisbon, Portugal \\ ${ }^{2}$ IDMEC, Instituto Superior Técnico, Universidade de Lisboa, Lisbon, Portugal \\ ${ }^{3}$ LNEC, Laboratório Nacional de Engenharia Civil, Lisbon, Portugal
}

\begin{abstract}
In this work, influence of geogrids on overall stabilization of railway tracks is studied numerically. It is expected that by geogrids implementation significant reduction in the downward propagation of stresses will be obtained, which consequently should assure more resilient long-term performance. In this first approach, however, only added value to the confinement level of the ballast layer is analysed, by evaluation of lateral deformations. A case study is related to a part of the Portuguese railway network. The track and the passing vehicle are modelled in commercial explicit dynamics software LS-DYNA. Firstly, the model is validated by comparison with experimental data. Then a fictitious scenario of the same track with a deteriorated region which is further rehabilitated by the geogrid placement is analysed. Different situations are compared in terms of lateral and vertical displacements at several levels, but for simplicity only rail deflections are shown here.
\end{abstract}

\section{Introduction}

European railway network is currently facing several challenges. Most of the existing lines are affected by aging factors, calling for urgent interventions, which is not in-line with ever growing demands on the network capacity and increased solicitations on durability and sustainability by implementation of heavy haul trains and other scenarios for which the lines have not been designed. Having in mind European Union regulations dictating shifting, as much as possible, air and road transport to rail, with environmental pretext, it can be concluded that efficient rehabilitation measures must be implemented. One of them can be accomplished with the help of new materials like geosynthetics and geotextiles. These materials have several beneficial properties and their use in new lines construction as well as in rehabilitation of existing lines is rapidly increasing.

In this work, influence of geogrids on overall stabilization of railway tracks is studied numerically. A typical ballasted railway track from Portuguese railway network is selected and modelled in commercial explicit dynamics software LS-DYNA. Full three-dimensional (3D) finite element (FE) model is used for the track, and the vehicle is simplified by moving two axles connected by steel beams representing the bogie.

\footnotetext{
* Corresponding author: zdim@,fct.unl.pt
} 
Due to the necessity of easily adaptable models of different geometry and material properties, it is not efficient to use LS-DYNA pre-processor and create each model directly there. Model creation was just coded in ANSYS Parametric Design Language (APDL). The first part of this program uses ANSYS pre-processing capabilities in model creation, meaning that by using a set of parameters defining all necessary geometry indicators the model is created including its FE mesh; the second part of the code re-writes the information about nodes and elements in form of LS-DYNA commands, adds the required properties, other specifications and commands for load application. Resulting file can be run directly in LS-DYNA solver and then result can be extracted in post-processing interface.

Firstly, the model is validated by comparison with experimental results. Then, a fictitious deterioration is introduced and rehabilitated by geogrid implementation. In these preliminary analyses it is assumed that interlocking mechanism is fully activated and therefore the geogrid uses its maximum capacity of stabilization. Materials are considered linear elastic and solutions are compared in terms of vertical and lateral displacements at several levels. In further developments time dependent non-linear behaviour of geomaterials will be implemented and several levels of interlocking efficiency will be introduced by friction mechanism between the layers and the geogrid. Long-term behaviour can be then analysed by modelling sequential passages of the vehicle.

\section{Model validation}

A typical ballasted railway track was selected in conformity with [1], where simplified twodimensional (2D) model was calibrated according to experimental results. Calibrated material values were used directly in the 3D FE model created in LS-DYNA. Thus, without any additional adjustment, very reasonable agreement between the experimental and numerical results was obtained.

Three layers in the superstructure in form of ballast, sub-ballast and capping layer are used. Foundation soil is modelled only by $3 \mathrm{~m}$ depth, but active soil depth is taken up to 7 $\mathrm{m}$. The width and length of the FE model are $10 \mathrm{~m}$ and $18 \mathrm{~m}$, respectively. Contrary to [1], only the homogeneous part of the track is used. All boundaries where soil is removed are covered by elastic springs to provide missing stiffness of the non-modelled soil, and dampers defining absorbing boundaries to prevent waves reflections. Absorbing boundaries are implemented in the default way available in LS-DYNA, which is based on developments made in [2]. Elastic springs on lateral boundaries are based on derivations in spherical coordinates and at the bottom equivalent springs are introduced, similarly as in [3]. At each node on these boundaries of the FE mesh, springs acting in three directions are implemented. In Figure 1, a detail of the FE mesh, as created in ANSYS is shown.

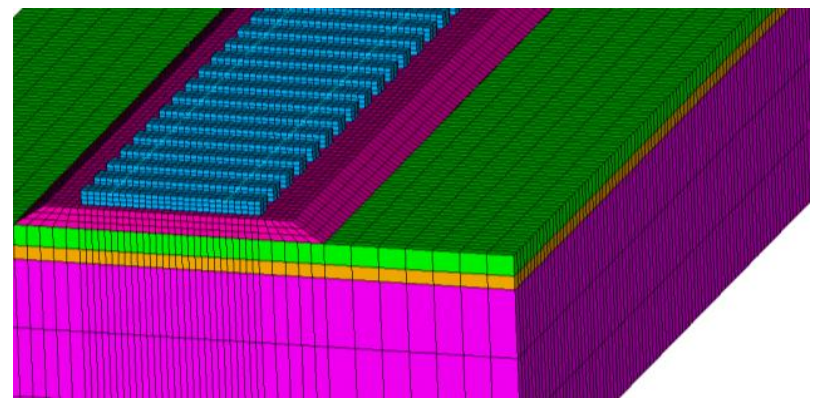

Figure 1. Detail of the FE model in ANSYS. 
Rails are approached by beam elements and rail pads by spring-dampers. The rail is connected to each sleeper by one spring-damper element in vertical direction, lateral rotation is prevented by imposing the same lateral displacement at the end nodes of the spring-damper. To avoid sharp concentrated peak on the sleeper in one point, vertical displacements of the sleeper are set equal below the rail.

As the load, locomotive of Alfa Pendular train passing by $61.1 \mathrm{~m} / \mathrm{s}$ as equivalent to 220 $\mathrm{km} / \mathrm{h}$ is used. For simplicity only the first bogie is modelled, which is sufficient for model validation and further analysis. Concentred masses of half axle mass are placed in contact with the rail and contact is modelled by default value of contact stiffness in LS-DYNA. Bogie is simplified by set of steel beams having the same mass as the full bogie. Wheels are connected to these beams by primary suspension. In location of concentrated masses, half axle loading forces are introduced. The graphs in Figure 2 represent the rail displacement measured at sleeper position at $12.3 \mathrm{~m}$ from the starting place. It is seen that the agreement with experimental results is quite reasonable, which validates the FE model.

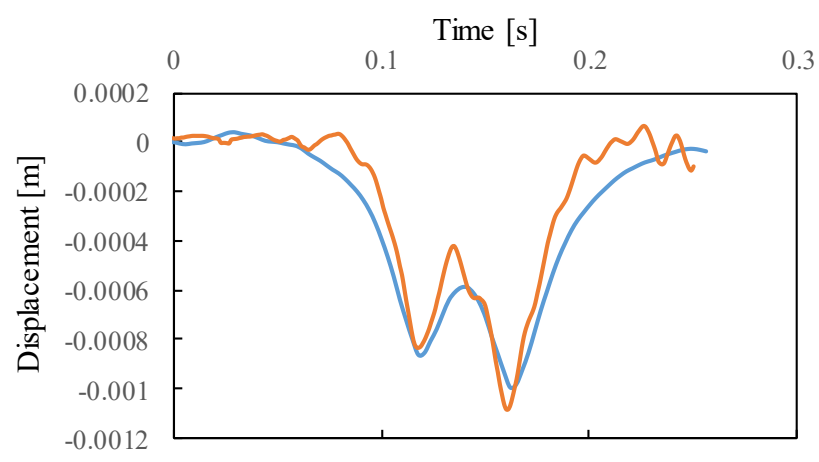

Figure 2. FE model validation: experimental (orange), numerical (blue).

Analyses that are performed on this research are quite fast due to explicit dynamics approach and in this context, it is useful to list several other advantage of software LSDYNA for similar investigations: (i) simple introduction of moving inertial objects; (i) no difficulties in contact introduction; and (iii) automatic specification of rail irregularities. All this can be simply introduced by command lines of *RAIL_TRAIN and *RAIL_TRACK.

After several analyses, it was concluded that the rails have a tendency of horizontal displacements, which can be harmful for the global track stability. In tested scenarios, both rails showed tendency to move laterally out. Therefore, fictitious scenarios with deteriorated parts were defined and effect on the stabilization by geogrid implementation was tested. This is described in further section.

\section{Tested scenarios and results obtained}

As a test solution, geogrid of typical values was implemented at the interface between the ballast and sub-ballast layer, over a central part of the model, ranging from 6 to $12 \mathrm{~m}$. In this first approach it is assumed that the interlocking mechanism is fully activated, thus geogrid was added by shell elements introduced directly at the common interface and overlaid on the solid element faces. As from technical sheets, the in-plane stiffness is defined by its effective value given by a secant modulus from force-per-length versus deformation graph, to get in-plane Young's modulus the secant modulus must be divided by the effective grid thickness, which is difficult to determine. Two homogenized 
thicknesses and the corresponding moduli were tested and no differences in results were found. This issue, however, still requires further analysis.

At first, the geogrid was placed in the original homogeneous track, secondly it was placed in the deteriorated track. Deterioration was defined by gradual decrease in Young's modulus to $40 \%, 50 \%$ and $60 \%$ of the original value in ballast, sub-ballast and capping layer, respectively. Affected regions started at 6.5, 7 and $7.5 \mathrm{~m}$ and ended at 11.5, 11 and $10.5 \mathrm{~m}$, respectively. Deteriorated regions can be seen in Figure 3.

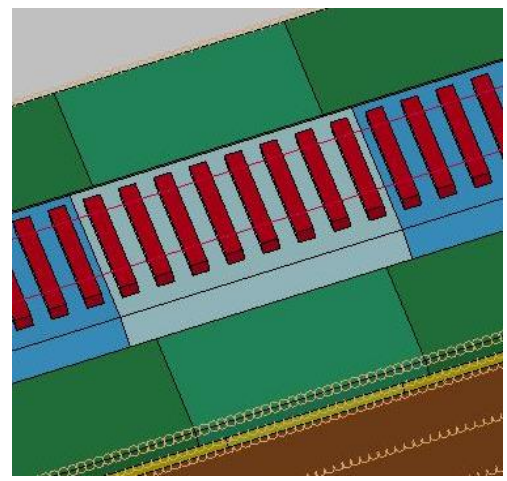

Figure 3. Detail of the FE model marking the deteriorated regions in LS-DYNA.

Thus, four scenarios were analysed, classified as: Case 1 - original homogeneous model, Case 2 - original model with geogrid, Case 3 - deteriorated model, Case 4 deteriorated model rehabilitated by geogrid. These four cases were compared in terms of rail displacements that are reported in the following figures. It was concluded that geogrid presence did not introduce noticeable differences in the rail vertical displacements. The reason is that there were not sufficiently high vertical displacements to activate geogrid membrane effect and in such a way increase the reinforcement in vertical direction. In Figure 4, the passage of the two wheels on the left rail and the final beam deflections of the four cases are shown. It is seen that the original configuration (Case 1) and the same configuration with geogrid (Case 2) are practically overlaid, as well as the deteriorated scenario (Case 3) and the rehabilitated one (Case 4).

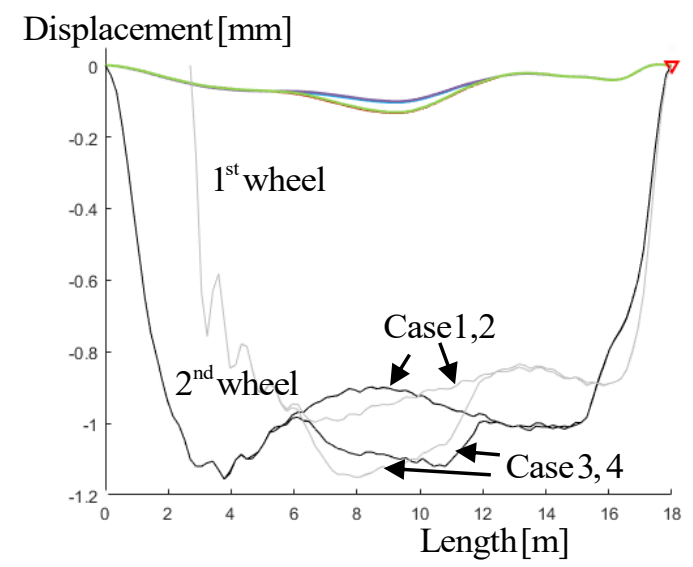

Figure 4. Vertical rail displacement: the passage of the two wheels $\left(1^{\text {st }}\right.$ wheel - grey, $2^{\text {nd }}$ wheel black) on the left rail and the final rail deflection in the four cases. 
Same situations for lateral displacements of the left rail look quite different, as some benefits are already observed. However, they are not visible while the wheels are passing on the tested regions, only on rail displacement posterior to the passage.

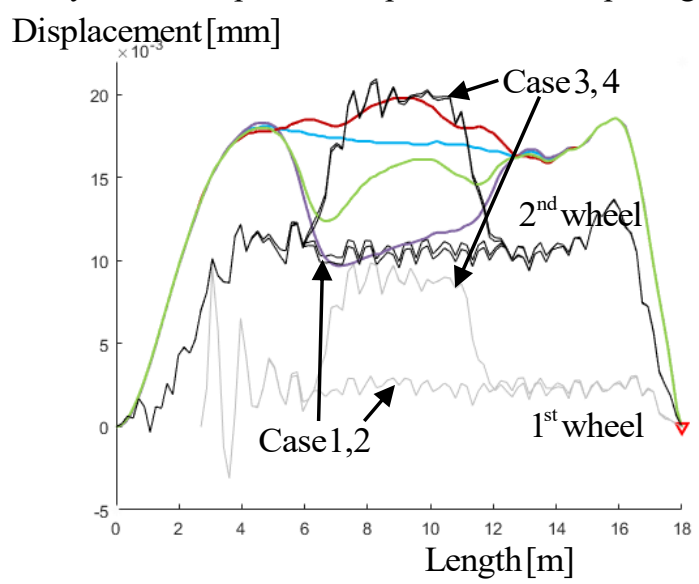

Figure 5. Horizontal rail displacement: the passage of the two wheels $\left(1^{\text {st }}\right.$ wheel - grey, $2^{\text {nd }}$ wheel black) on the left rail and the final rail deflection: Case 1 (blue), Case 2 (violet), Case 3 (red), Case 4 (green).

The fact is also clarified in Figure 6, where lateral displacements of the rail are shown for the four cases: part a) of the figure is related to the position when the second axle reaches the half of the model (and therefore the first wheel, which is $2.7 \mathrm{~m}$ ahead, has almost passed the tested region), and part b) when it reaches $90 \%$ of the model length, which also means that the first axle is already off the model. It is seen that in part a) the effect of the geogrid is only hardly seen.
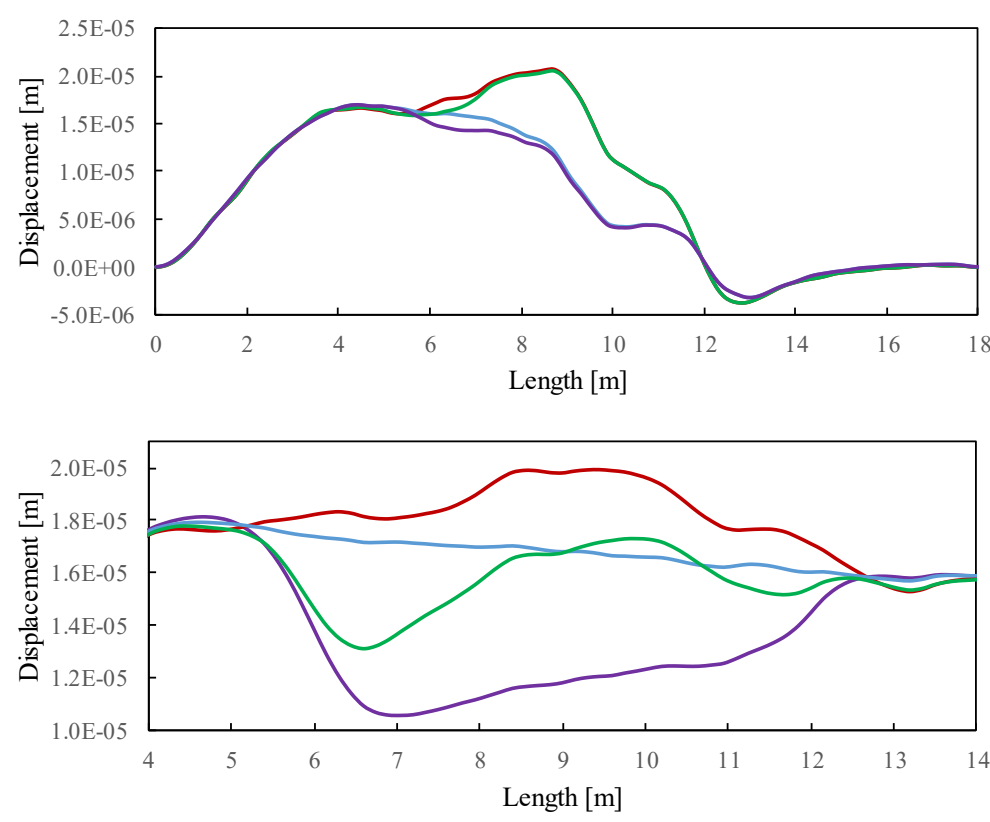

a)

b)

Figure 6. Lateral deflections of the left rail: Case 1 (blue), Case 2 (violet), Case 3 (red), Case 4 (green): a) $2^{\text {nd }}$ wheel at the middle, b) $2^{\text {nd }}$ wheel at $90 \%$ of the model (detail of the central region). 
To conclude this analysis, resultant force in lateral direction in the homogenized geogrid is shown in Figure 7 for Case 4 when the $2^{\text {nd }}$ axle is located in the middle of the model. It is seen that large traction is created at geogrid extremities. In Figure 7 user scale was forced in order to eliminate visualization of regions with localized values.

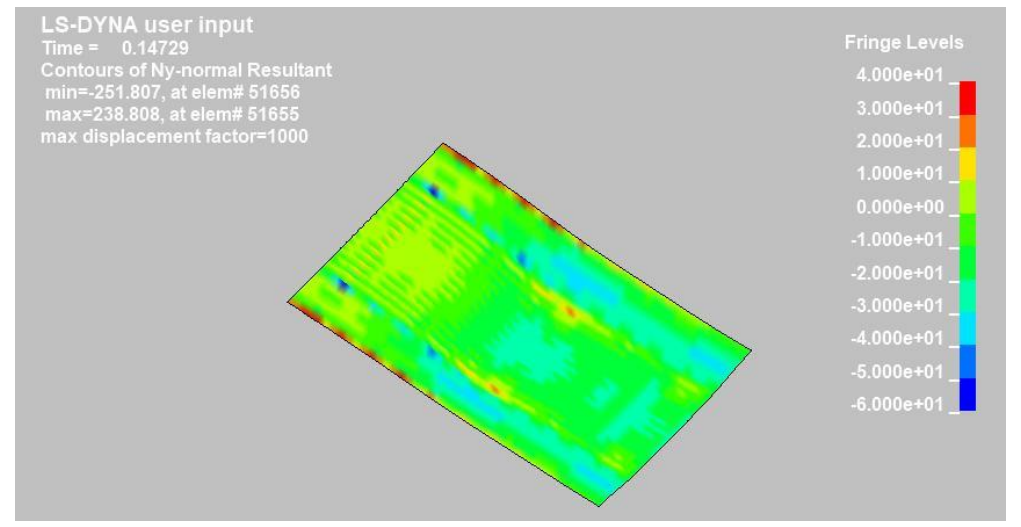

Figure 7. Lateral normal resultant force in the geogrid in Case 4 when $2^{\text {nd }}$ axle is in the middle.

\section{Conclusions}

In this paper benefits of geogrids implementation in rehabilitation of railway lines were analysed numerically. From functionalities that geogrids can offer, not all of them can be evaluated by analyses presented in this paper. For instance, layers separation could not be tackled, as, indeed in continues models the layers are naturally separated and do not mix. It was concluded that added value to vertical reinforcement is negligible, because only large displacements could activate the membrane effect. Regarding the vertical stiffness usually estimated as equivalent spring in series, geogrid contribution is naturally very low due to the low thickness and in-plane homogenized Young's modulus comparable with surrounding layers. Interlocking mechanism could not be evaluated and will be the subject of further research, but under the assumption of perfect interlocking, the geogrid was activated in in-plane directions and contributed significantly to the lateral stabilization by reducing the lateral displacements of the rail.

This work was partly supported by FCT, through IDMEC, under LAETA, project UID/EMS/50022/2013.

\section{References}

[1] A. Paixão, Transition zones in railway tracks - An experimental and numerical study on the structural behaviour, Ph.D. Thesis, Faculdade de Engenharia, Universidade do Porto (2014)

[2] J. Lysmer, R. Kuhlemeyer, J Eng Mech Div-ASCE 95(4), 859-877 (1969)

[3] A.F.S. Rodrigues, Viability and applicability of simplified models for dynamic analysis of railway tracks, Ph.D. Thesis, Faculdade de Ciências e Technologia, Nova University of Lisbon, Portugal (2017) 